\title{
Utilisation d'hydrolysats enzymatiques de caséine pour la croissance des bifidobactéries
}

\author{
M Proulx 1,2, SF Gauthier ${ }^{\star}$, D Roy $2^{\star \star}$ \\ 1 Université Laval, département des sciences et technologie des aliments, \\ centre de recherche en sciences et technologie du lait, Ste-Foy, Québec, G1K 7P4; \\ ${ }_{2}^{2}$ Agriculture Canada, centre de recherche et de développement sur les aliments, \\ groupe de recherche sur les bactéries lactiques, St-Hyacinthe, Québec, J2S 8E3, Canada
}

(Reçu le 18 novembre 1991; accepté le 6 mai 1992)

\begin{abstract}
Résumé - L'activité stimulante d'hydrolysats de caséine a été testée sur la croissance de 5 espèces du genre Bifidobacterium. Les enzymes alcalase, chymotrypsine et trypsine ont été utilisées pour générer les différents hydrolysats, lesquels ont été séparés par ultrafiltration sur membrane de type fibres creuses (seuil de coupure : $30000 \mathrm{Da}$ ). La croissance des bifidobactéries dans 3 milieux de culture semi-synthétiques ou synthétiques suggère que les peptides seraient une source d'azote plus adéquate que les acides aminés libres. Les milieux Garches et Norris ont été sélectionnés afin de comparer l'efficacité des différents hydrolysats de caséine ultrafiltrés à celle d'un mélange d'acides aminés et d'un hydrolysat commercial de caséine ( $\mathrm{N}-\mathrm{Z}$ Case), respectivement. Aucune stimulation de la croissance pour les espèces Bifidobacterium bifidum var pennsylvanicus et Bifidobacterium adolescentis n'a été observée, après $48 \mathrm{~h}$ d'incubation dans les milieux Garches et Norris contenant les hydrolysats ultrafiltrés. Bifidobacterium infantis présente une grande flexibilité au niveau de ses besoins en source d'azote. Dans le milieu Garches, on remarque une stimulation significative de l'activité acidifiante pour les espèces Bifidobacterium breve et Bifidobacterium longum. De façon générale, dans le milieu Norris, l'hydrolysat commercial N-Z Case assure les meilleures croissances aux 5 espèces bactériennes étudiées. Un effet stimulant du même ordre peut être obtenu chez $B$ infantis et $B$ breve avec les 3 autres types d'hydrolysats.
\end{abstract}

Bifidobacterium sp / hydrolysat de caséine / ultrafiltration / effet stimulant

Summary - Effect of casein hydrolysates on the growth of bifidobacteria. The growthpromotional activity of casein hydrolysates was tested for 5 species of the genus Bifidobacterium. Alcalase, chymotrypsin and trypsin were used to produce the casein hydrolysates which had been separated by ultrafiltration on a hollow fiber membrane (molecular weight cut-off: $30000 \mathrm{Da}$ ). Comparison of 3 semi-synthetic or synthetic media for cultivation of bifidobacteria indicated that peptides might be a preferable source of nitrogen to free amino acids. Garches and Norris media were selected to compare the efficiency of the different ultrafiltered hydrolysates to a mix of free amino acids (Garches) and to a commercial casein hydrolysate (Norris). After $48 \mathrm{~h}$ of growth, no difference appeared in terms of acidity between the ultrafiltered hydrolysates for the species Bifidobacterium bifi-

\footnotetext{
* Correspondance

** Tirés à part
} 
dum var pennsylvanicus and Bifidobacterium adolescentis. Bifidobacterium infantis showed a large flexibility regarding nitrogen requirement. In Garches medium, significant growth-promotional activity was obtained for the species Bifidobacterium breve and Bifidobacterium longum. In general, in the Norris medium commercial hydrolysate ( $N$-Z Case) allows better growth of the 5 bacterial species tested than ultrafiltered hydrolysates. A stimulating effect may be observed with ultrafiltered hydrolysates during growth of $\mathrm{B}$ infantis and $\mathrm{B}$ breve.

\section{Bifidobacterium sp / casein hydrolysate / ultrafiltration / growth enhancing factor}

\section{INTRODUCTION}

Au cours des dernières années, une troisième génération de laits fermentés est apparue sur le marché. Cette gamme de produits laitiers est préparée grâce à l'utilisation de bactéries lactiques d'origine intestinale. $\mathrm{Ce}$ sont les laits fermentés contenant des bifidobactéries qui connaissent actuellement le plus de succès de par le monde.

Dix espèces de bifidobactéries sur 28 sont d'origine humaine (Gavini et al, 1991). Les activités de ces bifides dans le tractus intestinal de l'homme peuvent se révéler importantes. L'ingestion chronique de laits fermentés contenant des bifidobactéries peut entraîner des modifications métaboliques au niveau intestinal (Marteau et al, 1990) tandis que l'ajout de facteurs bifidogènes dans les préparations alimentaires peut aider à stimuler la flore bifide du tractus intestinal (Tamura, 1983).

Par le passé, les études sur les facteurs bifidogènes ont mis en évidence l'effet stimulant des hydrolysats de protéines incluant la caséine (Raynaud et Bizzini, 1971). Jusqu'au début des années 1980 , ces facteurs n'ont été que très peu étudiés car la majorité des recherches a été limitée à l'espèce Bifidobacterium bifidum var pennsylvanicus. Celle-ci est un mutant, composant mineur de la microflore intestinale de l'homme, dont la croissance nécessite l'addition de lait humain. Les sucres dérivés de $\mathrm{N}$-acétylglucosamine
(Gyorgy et al, 1953) ainsi que certains glycopeptides (Bezkorovainy et al, 1979) retrouvés majoritairement dans le lait humain se sont avérés essentiels à la synthèse de sa paroi cellulaire (Veerkamp, 1969). Cependant, Kehagias et al (1977) ont obtenu une légère stimulation de la croissance de Bifidobacterium bifidum var pennsylvanicus lorsque de la caséine bovine, ayant subi une légère hydrolyse acide, était additionnée au milieu. Ils ont attribué cet effet stimulateur à des peptides issus de la dégradation de la caséine $\kappa$.

Des expériences sur les relations d'association existant entre les bifidobactéries et les lactobacilles, ont démontré que l'addition du filtrat cellulaire de Lactobacillus casei, après une croissance dans le lait, stimulait fortement la croissance et la production d'acide lactique et acétique de Bifidobacterium infantis et Bifidobacterium breve (Cheng et Nagasawa, 1984). Ce filtrat contenait des peptides de poids moléculaires situés entre 948 et $2319 \mathrm{Da}$.

D'autres travaux ont rapporté que les hydrolysats de caséine ainsi que les extraits de levure produisaient un effet stimulateur supérieur au lactosérum humain sur la croissance de différentes espèces de bifidobactéries autres que Bifidobacterium bifidum var pennsylvanicus (Poch et Bezkorovainy, 1988).

Pour mieux connaître les types de peptides responsables d'un effet stimulateur de croissance en fonction de l'espèce bactérienne, des hydrolysats de caséine contenant des peptides de taille et de na- 
ture différentes, générés par l'action de différentes enzymes protéolytiques (chymotrypsine, trypsine et alcalase) et fractionnés à l'aide de l'ultrafiltration, ont été ajoutés à des milieux de culture. Une comparaison des principaux milieux de culture utilisés jusqu'à présent dans les études relatives aux propriétés des hydrolysats de caséine sur la croissance des bifidobactéries a été faite. L'effet des différents hydrolysats ainsi que d'un hydrolysat commercial de caséine sur la croissance de 5 espèces du genre Bifidobacterium a été évalué par la mesure de leur activité acidifiante après $48 \mathrm{~h}$ d'incubation.

Cette étude fournira les renseignements nécessaires à la poursuite des travaux permettant de mettre en évidence que la nature et la taille des peptides peuvent avoir un effet marqué sur la croissance de certaines espèces de Bifidobacterium.

\section{MATÉRIEL ET MÉTHODES}

\section{Production des hydrolysats ultrafiltrés}

Le caséinate de sodium $(87,1 \%$ protéines sur base sèche) a été obtenu de ICN Pharmaceuticals Inc, Irvine CA. La chymotrypsine de pancréas bovin type II (EC 3.4.21.1), la trypsine de pancréas bovin type III-S (EC 3.4.21.4), les acides aminés et les vitamines provenaient de Sigma Chemical Co, St-Louis, MO. L'alcalase commerciale 2.5 L de Bacillus licheniformis a été fournie par la compagnie Novo Industri a/s Enzymes Division, Bagsvaerd, Danemark. Tous les autres produits étaient de qualité analytique.

Les fractions peptidiques ont été produites par l'ultrafiltration d'hydrolysats de caséine obtenus par l'action de l'alcalase, de la chymotrypsine ou de la trypsine selon le procédé mis au point par Turgeon et Gauthier (1990). Le caséinate de sodium a été hydrolysé en batch avec contrôle du pH ả 8,0 par l'addition de $\mathrm{NaOH} 4 \mathrm{~N}$. Les paramètres d'hydrolyse étaient les suivants : substrat, $60 \mathrm{~kg}$ de caséinate de sodium reconstitué à $3,5 \%$ de protéines; ratio enzyme- substrat, 1/200; $\mathrm{pH} 8,0$; température $40 \pm 1{ }^{\circ} \mathrm{C}$. Après $45 \mathrm{~min}$, les produits de l'hydrolyse ont été séparés (concentré $3 X$, diafiltré $2 X$ ) en utilisant des membranes de type fibres creuses (membrane Romicon HF1-43-PM30, Romicon Inc, Woburn, MA) dont le seuil de coupure était de $30000 \mathrm{Da}$. Le filtrat obtenu, appelé hydrolysat ultrafiltré, a été séché par lyophilisation.

\section{Méthodes d'analyse des hydrolysats}

Le degré d'hydrolyse en cours de réaction (45 $\mathrm{min})$ a été mesuré selon la méthode $\mathrm{pH}$-stat en utilisant 8,2 meq $^{-1}$ de protéines comme nombre total de liens peptidiques retrouvé dans la caséine (Adler-Nissen, 1977). Le contenu en protéines a été déterminé en triple selon la méthode de Kjeldahl décrite dans le document IDF 20A-Provisional Standards (1986) à l'aide d'un bloc de digestion Buchï 430 et d'une unité de distillation 321 (Brinkman Instruments, Montreal, $\mathrm{QC})$. Le degré d'hydrolyse des hydrolysats ultrafiltrés a été estimé en triple par la méthode spectrophotométrique OPA de dosage des groupements $\alpha$-aminés libres en utilisant la L-leucine comme standard (Church et al, 1983).

La distribution des poids moléculaires a été déterminée par chromatographie à haute performance par tamisage moléculaire (HPSEC, système LKB, colonne TSK-2000SW), selon la méthode de Vijayalakshmi et al (1986). Les standards protéiques étaient l'ovalbumine (42 $950 \mathrm{Da})$, la ribonucléase A (13 $710 \mathrm{Da})$, la polymyxine $\beta$-sulfate (1447 Da), la chaine $\beta$ de linsuline $(3480 \quad \mathrm{Da})$ et la L-leucyl-L-leucyl-Lleucine (358 Da). Les chromatogrammes ont été séparés en 3 zones de poids moléculaires différents (> $5000 \mathrm{Da}, 5000-2000 \mathrm{Da},<2000 \mathrm{Da}$ ) et chaque portion a été exprimée en pourcentage de la surface totale (Turgeon et Gauthier, 1990).

\section{Souches bactériennes et conditions de culture}

Cinq espèces du genre Bifidobacterium provenant de l'American Type Culture Collection (ATCC, Rockville, MD) ont été étudiées : Bifidobacterium bifidum var pennsylvanicus (ATCC 11863), Bifidobacterium infantis (ATCC 15697), Bifidobacterium breve (ATCC 15700), Bifidobac- 
terium longum (ATCC 15707) et Bifidobacterium adolescentis (ATCC 15703). L'identification des souches a été vérifiée par la présence de l'enzyme fructose-6-phosphate phosphokétolase (F6PPK; E.C. 4.1.2.22; Scardovi, 1981) à partir de bactéries perméabilisées en présence de Triton-X100 0,3\% (Chevalier et al, 1991) suivi d'un microtest de fermentation des sucres (Roy et Ward, 1990).

Chaque souche a été préalablement incubée sous atmosphère anaérobie contenant $5 \% \mathrm{CO}_{2}$, $10 \% \mathrm{H}_{2}$ et $85 \% \mathrm{~N}_{2}$ (anaerobic glove box; Format Scientific, Marietta, $\mathrm{OH}$ ) pendant $18 \mathrm{~h}$ à $37^{\circ} \mathrm{C}$ dans le milieu lactobacilli MRS broth (Difco Laboratories, Detroit, MI) (de Man et al, 1960) supplémenté de $0,05 \%$ de cystéine- $\mathrm{HCl}$. Les suspensions bactériennes ont été centrifugées à 8000 rpm pendant $15 \mathrm{~min}$. Les culots ont été lavés puis resuspendus dans un tampon Mcllvaine 100 $\mathrm{mmol} / \mathrm{I}\left(\mathrm{Na}_{2} \mathrm{HPO}_{4}\right.$ et acide citrique, $\left.\mathrm{pH} 6,0\right)$ pour obtenir des numérations initiales de $1 \times 10^{7} \mathrm{CFU} /$ $\mathrm{ml}$. Avant chaque groupe d'expériences, les milieux de culture ont été prè-équilibrés sous atmosphère anaérobie pendant $24 \mathrm{~h}$, afin d'éliminer l'oxygène dissous des milieux.

Les milieux Garches (Raynaud et Bizzini, 1971), Norris (Poupard et al, 1973) et Norris modifiè (Poch et Bezkorovainy, 1988) ont été préparés selon les directives de leurs auteurs. La composition des milieux utilisés est donnée au tableau I. Lors de la comparaison des milieux, les mesures de la densité optique, du $\mathrm{pH}$ et de l'acidité développée ont été effectuées aux temps $0,12,24,36,48,60$ et $72 \mathrm{~h}$. Dans la seconde partie de l'expérience, les hydrolysats ultrafiltrés ont remplacé, en concentration égale, les sources d'azote des milieux utilisés et les mesures ont été effectuées aux temps 0,12 , 24,36 et $48 \mathrm{~h}$. Des contrôles en aérobiose et en anaérobiose ont été effectués à chaque repiquage en étalant un échantillon des cultures sur gélose Lactobacilli MRS modifié (Roy et Ward, 1990) et Plate Count Agar (milieux MRS et PCA, Difco Laboratoires, Detroit, MI) à l'aide d'un écouvillon stérile.

\section{Méthodes d'analyse des cultures bactériennes}

La croissance bactérienne a été évaluée par spectrophotométrie (modèle 390 Spectrophotometer, Sequoia-Turner Corporation, Mountain
View, CA) à une longueur d'onde de $635 \mathrm{~nm}$ pour le milieu Garches et de $610 \mathrm{~nm}$ pour les milieux Norris et Norris modifié. Pour la détermination du $\mathrm{pH}$ et de l'acidité titrable, $5 \mathrm{ml}$ de suspension bactérienne ont été dilués dans $5 \mathrm{ml}$ d'eau déminéralisée. L'acidité titrable correspond à l'acidité développée, au cours de la croisssance des bactéries, exprimée en $\mathrm{mmol}$ de $\mathrm{NaOH}$ ajoutée pour atteindre le $\mathrm{pH}$ de 8,6 , auquel la valeur au temps 0 a été soustraite ( $\mathrm{pH}$-mètre modèle PHM 84 et titrimètre modèle TTT80, Radiometer, Copenhagen, DK).

\section{Analyses statistiques}

Toutes les courbes de croissance ont été réalisées en triple. L'effet des milieux sur l'acidité titrable à $48 \mathrm{~h}$ a été testé par une analyse de la variance. Celle-ci a été réalisée à l'aide de la procédure Proc GLM modèle général linéaire (SAS Institute 1989, version 6.06). La comparaison des hydrolysats ultrafiltrés a été effectuée par un test de comparaisons multiples sur les moyennes ajustées en corrigeant le niveau de confiance $(\alpha)$.

\section{RÉSULTATS}

\section{Production des hydrolysats ultrafiltrés}

La cinétique d'hydrolyse enzymatique du caséinate de sodium est présentée à la figure 1. La vitesse initiale d'hydrolyse est très rapide pour les enzymes trypsine et chymotrypsine par rapport à celle observée pour l'alcalase. Après 35-40 min, les hydrolyses trypsique et chymotrypsique sont maximales alors que la protéolyse par l'alcalase se poursuit à vitesse constante. Après $45 \mathrm{~min}$, le degré d'hydrolyse trypsique $(7,5 \%)$ est plus élevé que celui obtenu par l'alcalase et la chymotrypsine, lesquels sont comparables $(5,5 \%)$. Le tableau II présente les degrés d'hydrolyse des hydrolysats de caséine, obtenus après séparation par ultrafiltration (hydrolysats ultrafiltrés), 
Tableau I. Composition des milieux Garches, Norris et Norris modifié.

Composition of Garches, Norris and modified Norris media.

\begin{tabular}{|c|c|c|c|}
\hline \multirow{2}{*}{ Composés } & \multicolumn{3}{|c|}{ Types de milieu } \\
\hline & Garches & Norris & Norris modifié \\
\hline L-Acides aminés (g) & & & 21,00 \\
\hline Bacto casamino-acides (g) & 10,00 & & \\
\hline $\mathrm{N}-\mathrm{Z}$ Case $(\mathrm{g})$ & & 5,00 & \\
\hline Adénine $(\mathrm{g})$ & & 0,02 & 0,01 \\
\hline Alanine $(\mathrm{g})$ & & 0,20 & 0,20 \\
\hline Asparagine (g) & 5,00 & 0,10 & 0,10 \\
\hline Cystine $(\mathrm{g})$ & 0,20 & 0,20 & 0,20 \\
\hline Guanine $(\mathrm{g})$ & & 0,01 & 0,01 \\
\hline Tryptophane (g) & & 0,20 & 0,20 \\
\hline Uracile (g) & & 0,01 & 0,01 \\
\hline Xanthine $(\mathrm{g})$ & & 0,01 & 0,01 \\
\hline Acétate de sodium anhydre $(\mathrm{g})$ & 5,00 & 25,00 & 25,00 \\
\hline Chlorure de sodium (g) & & 0,01 & 0,01 \\
\hline Imidazole $(\mathrm{g})$ & & & 0,10 \\
\hline Lactose $(\mathrm{g})$ & 10,00 & 35,00 & 35,00 \\
\hline $\mathrm{K}_{2} \mathrm{PO}_{4}(\mathrm{~g})$ & 0,90 & 2,50 & 0,10 \\
\hline $\begin{array}{l}\mathrm{Na}_{2} \mathrm{HPO}_{4}(\mathrm{~g}) \\
\text { Sorbitan monooléate }\end{array}$ & 2,33 & & \\
\hline (Tween 80) (ml) & & 0,50 & \\
\hline Sulfate de fer $\cdot 7 \mathrm{H}_{2} \mathrm{O}(\mathrm{g})$ & & 0,01 & 0,04 \\
\hline Sulfate de magnésium $\bullet 7 \mathrm{H}_{2} \mathrm{O}(\mathrm{g})$ & 0,50 & 0,20 & 0,40 \\
\hline Sulfate de manganèse $\bullet \mathrm{H}_{2} \mathrm{O}(\mathrm{g})$ & & 0,01 & 0,03 \\
\hline Acide $p$-aminobenzoïque (mg) & 1,00 & 10,00 & 10,00 \\
\hline Acide ascorbique $(\mathrm{g})$ & & 1,00 & \\
\hline Acide folique (mg) & & 0,01 & 0,01 \\
\hline Acide nicotinique (mg) & & 0,60 & 0,60 \\
\hline Biotine $(\mu \mathrm{g})$ & 10,00 & 5,00 & 5,00 \\
\hline Pantothénate de calcium (mg) & 1,00 & 0,20 & 0,20 \\
\hline Pyridoxamine (mg) & & 1,20 & 1,20 \\
\hline Riboflavine (mg) & & 0,20 & 200,00 \\
\hline Thiamine (mg) & & 0,20 & 200,00 \\
\hline Volume final complété avec & & & \\
\hline de l'eau déminéralisée (I) & 1,00 & 1,00 & 1,00 \\
\hline
\end{tabular}

mesurés selon la méthode OPA. On note que ces degrés d'hydrolyse sont plus élevés que ceux obtenus par la méthode $\mathrm{pH}$ stat (fig 1). Cette différence s'explique par le fait que la teneur en $\mathrm{NH}_{3}$ libre de la caséine de départ n'a pas été soustraite de la valeur totale mesurée après hydrolyse. Comme observé à la figure 1, le degré d"hydrolyse de l'hydrolysat trypsique est supérieur (17\%) à celui de l'hydrolysat chymotrypsique (14\%). L'hydrolyse par l'alcalase, n'étant pas terminée après $45 \mathrm{~min}$, s'est 


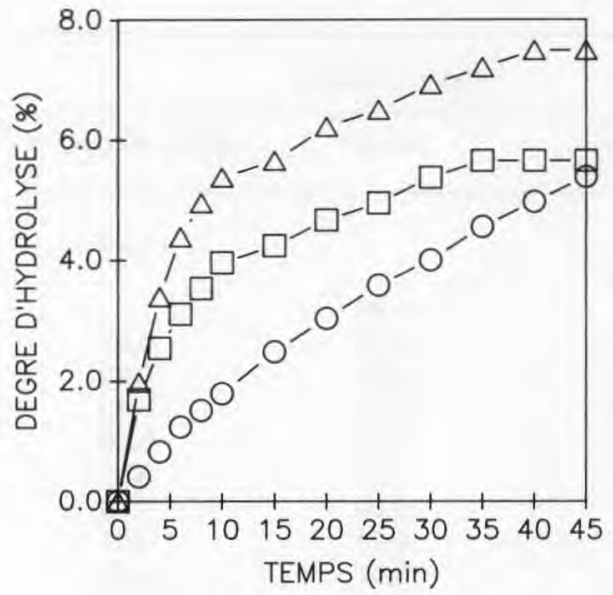

Fig 1. Cinétique d'hydrolyse du caséinate de sodium sous l'action de la trypsine $(\Delta)$, de la chymotrypsine $(\square)$ et de l'alcalase $(O)$. Le degré d'hydrolyse a été évalué par la méthode $\mathrm{pH}$-stat .(Adler-Nissen, 1977).

Hydrolysis kinetics of sodium caseinate with trypsin ( $(\Delta)$, chymotrypsin $(\square)$ and alcalase $(O)$. Degree of hydrolysis was estimated using the pH-stat method (Adler-Nissen, 1977). poursuivie en cours d'ultrafiltration. Ceci explique que le degré d'hydrolyse de cet hydrolysat $(18 \%)$ soit maintenant comparable à celui de l'hydrolysat trypsique $(17 \%)$. Enfin, l'hydrolysat enzymatique de caséine utilisé comme contrôle, le $\mathrm{N}-\mathrm{Z}$ Case, a un degré d'hydrolyse beaucoup plus élevé que tous les hydrolysats ultrafiltrés (43\%).

La distribution du poids moléculaire des hydrolysats caséiques reflète les degrés d'hydrolyse obtenus (tableau II). En effet, plus le degré d'hydrolyse est élevé, plus I'hydrolysat est riche en peptides de faibles poids moléculaires. Le $\mathrm{N}-\mathrm{Z}$ Case est essentiellement constitué de peptides de faibles poids moléculaires. L'alcalase et la trypsine ont eux aussi généré des hydrolysats ultrafiltrés contenant majoritairement des peptides de faibles poids moléculaires $(<2000 \mathrm{Da})$. Pour l'hydrolysat produit par l'action de l'alcalase, cette valeur atteint $95 \%$ pour un degré d'hydrolyse de $18 \%$ alors qu'elle est inférieure à $60 \%$ pour l'hydrolysat chymotrypsique dont le degré d'hydrolyse est de $14 \%$. De plus, I'hydrolysat chymotrypsique contient une propor-

Tableau II. Degré d'hydrolyse (OPA) et distribution des poids moléculaires (PM) des peptides contenus dans les hydrolysats ultrafiltrés.

Hydrolysis degree (OPA) and molecular weight (PM) distribution of peptides contained in ultrafiltered hydrolysates.

Contrôle

$N-Z$ Case

Hydrolysats ultrafiltrés

Alcalase Chymotrypsine Trypsine

$\begin{array}{lcclc}\text { Protéines (\%) } & 83,6 & 84,5 & 83,0 & 82,5 \\ \text { Degré d'hydrolyse (\%) } & 43,1 \pm 0,8 & 17,5 \pm 0,4 & 13,8 \pm 1,1 & 16,6 \pm 0,7 \\ P M>5000 \mathrm{Da}^{\mathrm{a}} & 0,0 & 0,01 & 24,3 & 4,0 \\ 5000 \mathrm{Da}>\mathrm{PM}>2000 \mathrm{Da} & 0,4 & 5,2 & 16,7 & 11,3 \\ \mathrm{PM}<2000 \mathrm{Da} & 99,6 & 94,8 & 59,0 & 84,7\end{array}$

\footnotetext{
a La surface totale du chromatogramme est intégrée puis divisée en 3 zones de poids moléculaires différents. Chaque zone représente le pourcentage de surface totale (Turgeon et Gauthier, 1990).

The total surface of the chromatogram is included then divided into 3 zones of different molecular weights. Each zone represents the percentage of the total area (Turgeon and Gauthier, 1990).
} 
tion importante $(23 \%)$ de peptides de poids moléculaires élevés (> $5000 \mathrm{Da}$ ) contrairement aux hydrolysats produits par les 2 autres enzymes.

\section{Sélection d'un milieu de culture}

Les figures 2 et 3 montrent que la croissance et l'activité acidifiante de $B$ bifidum var pennsylvanicus sont presque nulles

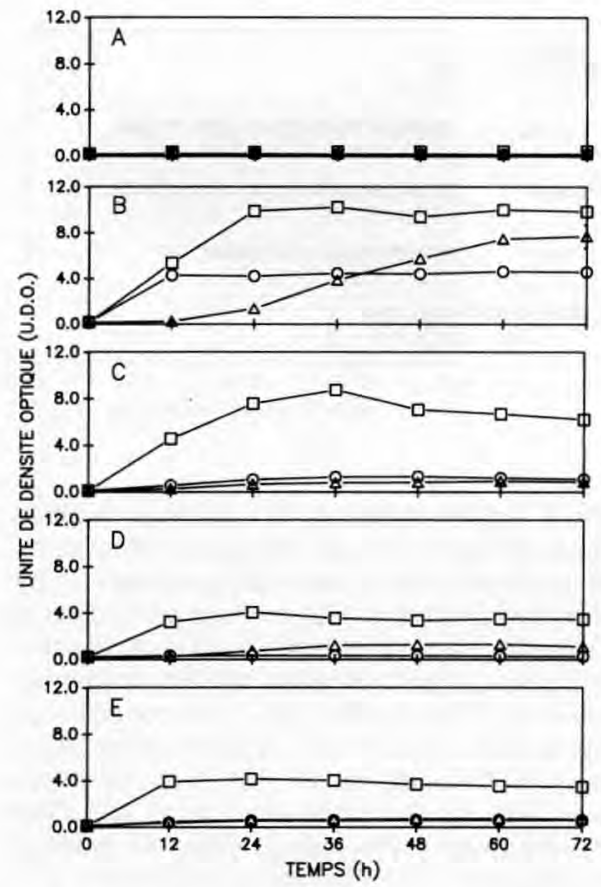

Fig 2. Croissance de $B$ bifidum var pensylvanicus (A), B infantis (B), B breve (C), B longum (D) et $B$ adolescentis (E) dans les milieux Garches (O), Norris $(\square)$ et Norris modifié $(\Delta)$. Les valeurs présentées sont des moyennes \pm erreurs standard $(P<0,05)$ de courbes de croissance effectuées en triple.

Growth of $B$ bifidum var pennsylvanicus $(A), B$ infantis $(B)$, B breve $(C)$, B longum $(D)$ and $B$ adolescentis $(E)$ in Garches $(O)$, Norris $(\square)$ and modified Norris media $(\Delta)$. Values are means $\pm S E$ $(\mathrm{P}<0.05)$ of growth curves performed in triplicate.

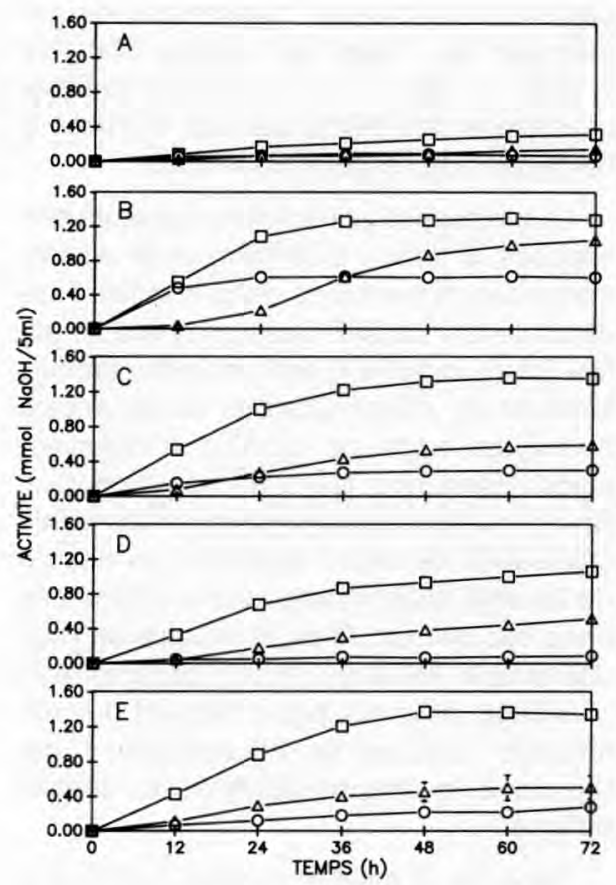

Fig 3. Activité acidifante de $B$ bifidum var pennsylvanicus (A), $B$ infantis (B), B breve (C), B longum (D) et $B$ adolescentis (E) dans les milieux Garches $(O)$, Norris $(\square)$ et Norris modifié $(\Delta)$. Les valeurs présentées sont des moyennes \pm erreurs standards $(P<0,05)$ de courbes de croissance effectuées en triple.

Acid production activity of $\mathrm{B}$ bifidum var pennsylvanicus $(A), B$ infantis $(B), B$ breve $(C)$, B longum $(D)$ and $B$ adolescentis $(E)$ in Garches $(O)$, Norris ( $\square$ ) and modified Norris media ( $\Delta$ ). Values are means $\pm S E(P<0.05)$ of growth curves performed in triplicate.

dans les 3 milieux de culture (fig $2 A$ et $3 A$ ). L'absence de facteur bifidogène de nature glucidique permet d'expliquer l'inactivité de cette souche.

La croissance et l'activité acidifiante des 4 autres espèces de Bifidobacterium sp sont supérieures dans le milieu Norris 
contenant l'hydrolysat commercial de caséine, le N-Z Case. Les milieux Garches et Norris modifié n'ont permis qu'une faible croissance des bifidobactéries étudiées à l'exception de l'espèce $B$ infantis.

La croissance et l'activité acidifiante des espèces $B$ breve, $B$ longum et $B$ adolescentis sont supérieures dans le milieu Norris contenant du $\mathrm{N}-\mathrm{Z}$ Case comparé au milieu Norris modifié à base d'acides aminés libres et au milieu Garches contenant un hydrolysat acide de caséine (Casaminoacids). Bien que les milieux Garches et Norris modifié n'aient permis qu'une faible croissance de ces 3 espèces, la mesure de l'acidité titrable indique des différences entre ces milieux. Ainsi, la mesure de l'acidité titrable se révèle être le paramètre le plus fiable pour comparer l'activité des différentes espèces de bifidobactéries par rapport à la détermination de la densité optique.

Dans les 3 milieux étudiés, l'espèce $B$ infantis présente un comportement différent des autres espèces. La croissance et l'activité acidifiante de $B$ infantis dans le milieu Garches sont comparables à celles obtenues avec le milieu Norris au cours des 12 premières heures d'incubation (fig 2B et 3B). Dans le milieu Garches, l'acidité titrable atteint une valeur approximative de $0,60 \mathrm{mmol}$ par $5 \mathrm{ml}$ de culture après $24 \mathrm{~h}$ d'incubation et se maintient à cette valeur au cours de la période de $72 \mathrm{~h}$ étudiée. L'épuisement du lactose dans le milieu Garches serait responsable de l'arrêt de la croissance.

\section{Comparaison des activités en présence des hydrolysats de caséine}

À la suite des résultats obtenus, la mesure de l'acidité titrable a été retenue pour comparer les différents hydrolysats de caséine obtenus après ultrafiltration. La figure 4
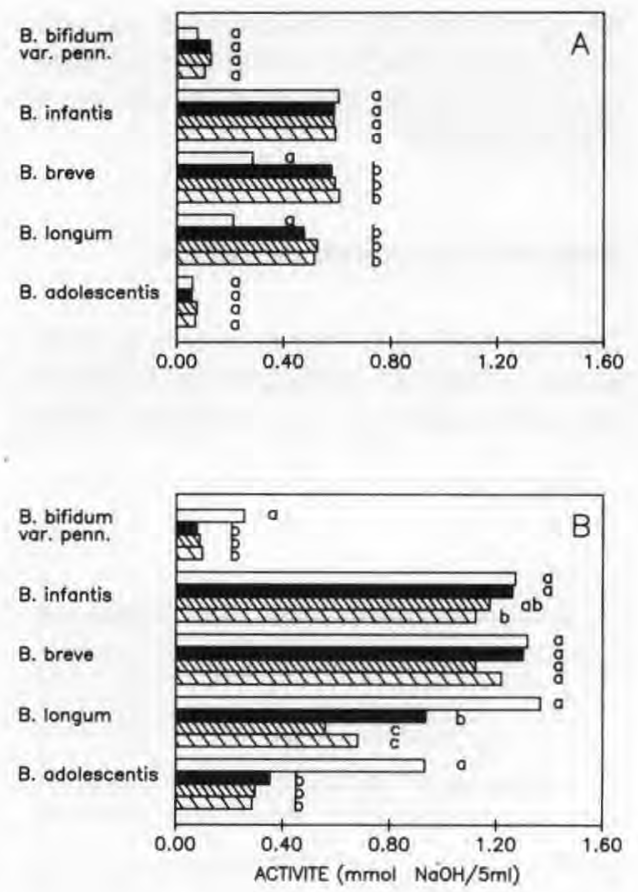

Fig 4. Activité acidifiante de 5 espèces de bifidobactéries après $48 \mathrm{~h}$ de croissance dans les milieux Garches (A) et Norris (B) contenant les hydrolysats ultrafiltrés (HU) produits par l'action de l'alcalase, de la chymotrypsine et de la trypsine. Contrôle ( $\square$ ); HU alcalase (四); HU chymotrypsine $(\mathbb{Q})$; HU trypsine $(\mathbb{\nabla})$. Les contrôles ont été faits en ajoutant des Casamino-acids (A) ou du $\mathrm{N}-\mathrm{Z}$ Case (B) tandis que dans les essais avec $\mathrm{HU}$, les Casamino-acids ou le $\mathrm{N}-\mathrm{Z}$ Case étaient absents. Des lettres différentes indiquent la présence de différences significatives $(P<$ $0,05)$. Les valeurs présentées sont des moyennes d'expériences effectuées en triple.

Acid production activity of 5 bifidobacteria species after $48 \mathrm{~h}$ of growth in Garches (A) and Norris (B) media containing ultrafiltered hydrolyzates (UH) produced with alcalase, chymotrypsin and trypsin. Control ( $\square$ ); alcalase

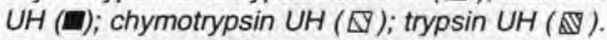
Controls were performed by adding casaminoacids (A) or $\mathrm{N}-\mathrm{Z}$ case (B) whereas in experiments with $U H$, casamino-acids or $N-Z$ case were omitted. Different letters indicate significant differences $(P<0.05)$. Values are means $(\mathrm{P}<0.05)$ of experiments performed in triplicate. 
présente l'activité acidifiante des bifidobactéries développée pendant $48 \mathrm{~h}$ d'incubation dans les milieux Garches $(A)$ at Norris (B) dans lesquels les Casamino-acids ou le N-Z Case (contrôle excepté) ont été remplacés par les hydrolysats ultrafiltrés de l'alcalase, de la chymotrypsine ou de la trypsine.

De façon générale, dans le milieu Norris, I'hydrolysat commercial de caséine est la source d'azote la plus appropriée (fig 4B). Aucune stimulation de l'activité acidifiante pour l'espèce $B$ bifidum var pennsylvanicus n'a toutefois été observée dans les milieux Garches et Norris.

Dans l'ensemble, les milieux Garches et Norris contenant les hydrolysats ultrafiltrés ont cependant permis une activité acidifiante comparable aux contrôles, contenant des Casamino-acide ou du N-Z Case, pour l'espèce $B$ infantis. Cette espèce a manifesté une activité acidifiante limitée d'une part, par le $\mathrm{pH}$ acide résultant d'une forte croissance dans le milieu Norris (données non présentées) et d'autre part, par la faible concentration en lactose du milieu Garches (tableau I). Comme il a été observé lors de la comparaison des milieux, il y a utilisation complète du lactose dans le milieu Garches. $B$ infantis semble donc avoir peu d'exigence pour ses sources d'azote et peut utiliser une vaste gamme de peptides pour sa croissance.

Les espèces $B$ breve et $B$ longum ont été stimulées par les hydrolysats ultrafiltrés de l'alcalase, de la chymotrypsine et de la trypsine ajoutés au milieu Garches. Pour B breve comme pour B infantis, les hydrolysats ultrafiltrés ajoutés au milieu Norris ont permis une activité acidifiante égale à celle obtenue avec le $\mathrm{N}-\mathrm{Z}$ Case. Toutefois, ces mêmes hydrolysats n'induisent qu'une acidification nettement inférieure chez $B$ longum et $B$ adolescentis.

\section{DISCUSSION}

La spécificité des enzymes utilisées a influencé la cinétique d'hydrolyse de la caséine de même que la répartition du poids moléculaire des peptides produits. La trypsine est une endopeptidase spécifique aux acides aminés basiques, arginine et lysine. La chymotrypsine est aussi une endopeptidase mais sa spécificité se situe de préférence au niveau des acides aminés aromatiques tels que la phénylalanine, la tyrosine et le tryptophane. L'hydrolyse par la trypsine s'est révélée plus rapide et plus élevée qu'avec la chymotrypsine puisque les résidus basiques sont plus accessibles (en surface de la conformation) et en plus grand nombre dans les caséines que les résidus aromatiques de nature également plus hydrophobe.

Comme la chymotrypsine, l'alcalase est une endopeptidase qui présente une préférence pour les acides aminés aromatiques. Elle peut aussi hydrolyser des liens constitués des acides aminés cibles de la trypsine (Ottensen et Svendsen, 1970). La spécificité plus large de l'alcalase permet donc d'expliquer le plus grand pourcentage de peptides de faibles poids moléculaires $(<2000 \mathrm{Da})$ contenu dans son hydrolysat ultrafiltré.

Les résultats de cette étude sur la croissance des bifidobactéries démontrent que l'espèce Bifidobacterium bifidum var pennsylvanicus se démarque des autres espèces du genre Bifidobacterium puiqu'elle ne peut être stimulée en présence d'hydrolysats de caséine bovine. Cette espèce a servi, jusqu'à maintenant, de culture témoin dans la majorité des études sur les facteurs bifidogènes (Gyorgy et al, 1953, Gyorgy et Rose, 1955; Nichols et Bezkorovainy, 1974; Kehagias et al, 1977; Bezkorovainy et al, 1979; Bezkorovainy et Topouzian, 1981). II a été démontré que cette souche est en fait un mutant, rare 
composant de l'intestin des nouveau-nés, nécessitant des sucres dérivés du Nacétylglucosamine (Gyorgy et al, 1953) et de certains glycopeptides retrouvés majoritairement dans le lait humain (Bezkorovainy et al, 1979). Ces composés sont essentiels à la synthèse de sa paroi cellulaire (Veerkamp, 1969).

Toutes les espèces étudiées ont démontré une activité acidifiante supérieure dans le milieu semi-synthétique Norris contenant du N-Z Case (hydrolysat commercial de caséine), comparativement à celle observée dans le milieu Norris synthétique contenant 21 acides aminés libres. Les peptides semblent donc être une source d'azote plus adéquate pour la croissance des bifidobactéries. Ces résultats sont en accord avec ceux obtenus par Poch et Bezkorovainy (1988).

Parmi les espèces étudiées, $B$ infantis a présenté la plus grande flexibilité au niveau de ses besoins en source d'azote s'accommodant autant de peptides que d'acides aminés libres. Cette souche est la principale bactérie retrouvée dans le tube intestinal des nouveau-nés. Chez $B$ breve, $B$ longum et $B$ adolescentis, il semble que l'absence de certaines vitamines ait été un facteur limitant pour leur croissance. Comparativement à $B$ infantis, ces dernières ne sont pas des espèces hautement productrices et accumulatrices de vitamines telles que la thiamine, l'acide folique et l'acide nicotinique (Deguchi et al, 1985). Ceci peut expliquer la meilleure performance du milieu Norris modifié sur le milieu Garches dépourvu de ces vitamines (tableau I). Ce besoin en vitamines est encore plus évident pour $B$ adolescentis.

En comparaison à un hydrolysat acide de caséine (Garches), les hydrolysats enzymatiques ultrafiltrés ont permis une bonne activité acidifiante de $B$ breve et de $B$ longum tandis que celle de $B$ infantis est peu influencée (fig 4a). Dans le milieu Nor- ris, l'activité acidifiante de $B$ bifidum var pennsylvanicus, $B$ longum et $B$ adolescentis en présence des hydrolysats ultrafiltrés est inférieure à celle présente en milieu Norris contrôle contenant du $\mathrm{N}-\mathrm{Z}$ Case contrairement à ce que l'on observe avec les espèces $B$ infantis et $B$ breve (fig 4B). Ces résultats suggèrent que la spécificité de l'enzyme utilisée pour la production de l'hydrolysat ainsi que le degré d'hydrolyse obtenus sont importants, car la nature et la taille des peptides générés par un type d'enzyme ne sont pas nécessairement appropriées à la croissance de toutes les espèces de bifidobactéries.

D'ailleurs, l'hydrolysat produit par l'action de l'alcalase contenant $95 \%$ de peptides de poids moléculaires inférieurs à 2000 Da est celui, excepté le N-Z Case, qui permet la meilleure activité acidifiante des espèces $B$ infantis, $B$ breve, $B$ longum et $B$ adolescentis. Ces résultats concordent avec ceux de Cheng et Nagasawa (1984), qui avaient démontré que les peptides de poids moléculaires compris entre 948 et $2319 \mathrm{Da}$, contenus dans le filtrat cellulaire de Lactobacillus casei, stimulaient la croissance de $B$ infantis et de $B$ breve. L'effet stimulateur de croissance du $\mathrm{N}-\mathrm{Z}$ Case et de l'alcalase pourrait être relié d'une part, à la taille des peptides et, d'autre part, à la nature des peptides générés.

De notre étude, il se dégage clairement que les hydrolysats de caséine obtenus par hydrolyse enzymatique, en particulier le $\mathrm{N}-\mathrm{Z}$ Case, sont des sources d'azote plus adéquates pour la majorité des bifidobactéries étudiées que les acides aminés. De façon générale, dans le milieu Norris, le N$Z$ Case assure les meilleures croissances aux 5 espèces bactériennes étudiées. Un effet stimulant du même ordre peut être obtenu chez $B$ infantis et $B$ breve avec les 3 autres types d'hydrolysat. Le poids moléculaire des peptides contenus dans les hydrolysats enzymatiques semble être un 
facteur important. Pour confirmer l'importance de ce facteur, une étude plus approfondie des peptides contenus dans les différents hydrolysats pourra nous aider à mieux élucider les besoins en peptides des bactéries du genre Bifidobacterium communément utilisées dans les préparations alimentaires.

\section{REMERCIEMENTS}

Nous tenons à remercier $\mathrm{C}$ Bard et $\mathrm{F}$ Lépine pour leur aide technique ainsi que $\mathrm{N}$ Rodrigue pour sa collaboration au traitement statistique des données. Nos remerciements s'adressent également à L Blanchette, J Gagné, L Savoie et $P$ Ward pour les conseils et remarques pertinentes lors de l'exécution des plans expérimentaux. Contribution no 251 du Centre de recherche et de développement sur les aliments.

\section{RÉFÉRENCES}

Adler-Nissen $J$ (1977) Enzymatic hydrolysis of food proteins. Process Biochem 12, 18-19, 22-23, 32

Bezkorovainy A, Topouzian N (1981) Bifidobacterium bifidus var pennsylvanicus growth promoting activity of human milk casein and its derivatives. Int J Biochem 13, 585-590

Bezkorovainy A, Grohlich D, Nichols JH (1979) Isolation of a glycopolypeptide fraction with Lactobacillus bifidus subspecies pennsylvanicus growth promoting activity from whole human milk casein. Am J Clin Nutr 32, 14281432

Cheng CC, Nagasawa T (1984) Effect of peptides and amino acids produced by Lactobacillus casei in milk on the acid production of bifidobacteria. Jpn J Zootech Sci 55, 339349

Chevalier P, Roy D, Savoie L (1991) X- $\alpha$-Galbased medium for simultaneous enunieration of bifidobacteria and lactic acid bacteria in milk. J Microbiol Methods 13, 75-83

Church FC, Swaisgood HE, Porter DH, Catignani GL (1983) Spectrophotometric assay using o-phthaldialdehyde for determination of pro- teolysis in milk and isolated milk proteins. J Dairy Sci 66, 1219-1227

Deguchi Y, Morishita T, Mutai M (1985) Comparative studies on synthesis of water-soluble vitamins among human species of bifidobacteria. Agric Biol Chem 49, 13-19

de Man JC, Rogosa M, Sharpe E (1960) A medium for cultivation of lactobacilli. J Appl Bacteriol 23, 130-135

Gavini F, Pourcher AM, Neut C, Monget D, Romond C, Oger C, Izard D (1991) Phenotypic differentiation of bifidobacteria of human and animal origins. Int J Syst Bacteriol 41, 548-552

Gyorgy P, Rose CS (1955) Further observations on the metabolic requirements of Lactobacillus bifidus var pennsylvanicus. $J$ Bacteriol $69,483-490$

Gyorgy P, Norris RF, Rose CS (1953) Bifidus factor. I. A variant of Lactobacillus bifidus requiring a special growth factor. Arch Biochem Biophys 48, 193-201

Kehagias C, Jao YC, Mikolajcik EM, Hansen PMT (1977) Growth response of Bifidobacterium bifidum to a hydrolytic product isolated from bovine casein. J Food Sci 42, 146-150

Marteau P, Pochart P, Flourié B, Pellier $P$, Santos L, Desjeux JF, Rambaud JC (1990) Effect of chronic ingestion of fermented dairy product containing Lactobacillus acidophilus and Bifidobacterium bifidum on metabolic activities of the colonic flora in humans. Am J Clin Nutr 52, 685-688

Nichols JH, Bezkorovainy A (1974) Human colostral whey $M-1$ glycoproteins and their $L b i$ fidus var pennsylvanicus growth promoting activities. Life Sci 14, 967-976

Ottensen M, Svendsen I (1970) The subtilisins. In: Methods in enzymology, vol XIX. Proteolytic enzymes (Perlmann GE, Lorand L, eds) Acad Press, New York, 199-215

Poch M, Bezkorovainy A (1988) Growthenhancing supplements for various species of the genus Bifidobacterium. J Dairy Sci 71, 3214-3221

Pourpard JA, Husain I, Norris RF (1973) Biology of the bifidobacteria, Bacteriol Rev 37, 136-165

Raynaud M, Bizzini B (1971) Purification et propriétés du facteur bifidus 2. Ann Nutr Aliment 25, 209-223

Roy D, Ward P (1990) Evaluation of rapid methods for differentiation of Bifidobacterium species. J Appl Bacteriol 69, 739-749 
SAS Institute Inc (1989) SAS ${ }^{(1)}$ user's guide: statistics version 6.06. SAS Institute Inc, Cary NC,

Scardovi V (1981) The genus Bifidobacterium. In: The Prokaryotes. A handbook on habitats, isolation and identification of bacteria (Starr MP, Stolp H, Truper HG, Balows A, Schlegel HG, eds) A Balows et HG Schlegel, Springer, New York

Tamura Z (1983) Nutriology of bifidobacteria. Bifidobacteria Microflora 2, 3-16
Turgeon SL, Gauthier SF (1990) Whey peptide fractions obtained with a two-step ultrafiltration process: production and characterization. J Food Sci 55, 106-110, 157

Veerkamp JH (1969) Uptake and metabolism of derivatives of 2-deoxy-2-amino-D-glucose in Bifidobacterium bifidum var pennsylvanicus. Arch Biochem Biophys 129, 248-256

Vijayalakshmi M, Lemieux L, Amiot J (1986) HPLC analytical separation of peptides according to their molecular weight. $J$ Liq Chromatogr 9, 3559-3576 